\title{
Challenges in diagnosing brain death
}

\author{
Martin R. Lessard, MD • Jacques G. Brochu, MD
}

Published online: 29 July 2010

(C) Canadian Anesthesiologists' Society 2010

Recognizing that the nature of the practice of clinical medicine involves areas of uncertainty, death is one diagnosis that doctors must not be allowed to miss. The concept of brain death, which originates from the Harvard Criteria, ${ }^{1}$ has allowed development of cadaveric organ transplantation programs. Over the years, the definition of brain death and the criteria for the neurological determination of death (NDD) have been updated. ${ }^{2-4}$ The Universal Determination of Death Act provides a definition of death but leaves the determination process to accepted medical standards (national, provincial, or even local), which can entail a high variability. ${ }^{2,5}$ In April 2003, in response to a perceived need in the general medical and critical care community, the Canadian Council for Donation and Transplantation (CCDT) held a national forum to revisit the process of declaring brain death and to propose updated recommendations. ${ }^{6}$ These are the current guidelines used by organ donation and transplantation organizations across Canada. ${ }^{7}$ The CCDT recommendations have the virtue of having brought uniformity to the process of NDD. Still, several of its recommendations are somewhat vague and open the door to clinical judgement and interpretation, a situation that is highly undesirable when facing potential organ donation.

In this issue of the Journal, Roberts et al. report two cases where experienced physicians faced challenges in the clinical NDD. ${ }^{8}$ In both cases, it appears that several of the criteria for NDD were either misinterpreted or overlooked.

M. R. Lessard, MD ( $\varangle) \cdot$ J. G. Brochu, MD

Department of Anesthesia and Critical Care, Centre hospitalier affilié universitaire de Québec, Université Laval, 1401, 18e rue, Québec, QC G1J 1Z4, Canada

e-mail: martin.lessard@anr.ulaval.ca
Although they are usually not reported in the medical literature, similar incidents occur more than occasionally. This is somewhat disturbing. The existence of cadaveric organ donation and transplantation programs relies on the Dead Donor Rule that dictates that a patient's death must be confirmed before any attempt at organ preservation or harvesting for the purpose of transplantation. ${ }^{9}$ Before a patient is dead, ethical principles mandate that medical care must be directed toward the sole and only benefit of this patient, even in a situation that could be perceived as "almost dead". Brain death is a complex concept that is very difficult for the general public to understand. ${ }^{10,11}$ Patients and their families place their trust in their treating physician. ${ }^{12}$ In organ donation, they trust that physicians will strictly abide by the Dead Donor Rule. Therefore, the process for NDD must be watertight.

The CCDT recommendations define neurological death as the irreversible loss of all brain stem functions. ${ }^{7}$ The minimum criteria for clinical NDD are: 1) an established etiology capable of causing neurological death in the absence of reversible conditions capable of mimicking neurological death; 2) a deep unresponsive coma with bilateral absence of motor responses (excluding spinal reflexes); 3) absent brain stem reflexes, as defined by absent gag and cough reflexes, and the bilateral absence of corneal responses, pupillary responses to light (with pupils at mid-size or greater), and vestibulo-ocular responses; 4) absent respiratory effort based on the apnea test; and 5) absence of any confounding factors. ${ }^{7}$ Minimum means that at least all of these criteria must be met for clinical NDD.

Confusion may arise regarding the first criterion. An etiology "capable of causing" neurological death is not the same as "sufficient to account for" the absence of clinical signs of brain function. For example, brain trauma is an 
etiology capable of causing brain death, but obviously not all brain traumas will end with neurological death. Patients have survived, with a good functional outcome, a severe acute brain injury that might have resulted in brain death. ${ }^{13,14}$ This is why the CCDT recommendations specifying that "There must be definite clinical or neuroimaging evidence of an acute central nervous system (CNS) event consistent with the irreversible loss of neurological function." ${ }^{77}$ There must be an absolute congruence between the diagnosis entity causing the brain damage, its severity, and the clinical neurological state of the patient. In that respect, diagnostic neuro-imaging examinations (e.g., computed tomography [CT]-scan, magnetic resonance) are most useful, and their careful interpretation is critical. If there is any doubt regarding the etiology or its congruence with the clinical state, physicians should not proceed with NDD.

There may also be some confusion regarding the interpretation and conduct of the apnea test. Spontaneous resumption of breathing has been observed following a positive apnea test. ${ }^{8}$ A positive apnea test is not, in itself, sufficient to declare neurological death. Rather, it is the last step of brain stem function testing, and it should be conducted only after confirmation that all other brainstem reflexes are absent and all other criteria for NDD have been met. There have been a number of publications on the technique as well as on the safety and complications of the apnea test. ${ }^{15-17}$ A positive apnea test is defined as the absence of respiratory effort following disconnection from the ventilator, with the $\mathrm{PaCO}_{2}$ rising above $60 \mathrm{mmHg}$ (and $\geq 20 \mathrm{mmHg}$ above the pre-apnea test level) and $\mathrm{pH}$ below 7.28. ${ }^{7}$ Unfortunately, guidelines do not specify how long the patient must be observed. Admittedly, it is unknown how long the hypercapnic challenge must last to ensure the absence of respiratory centre function, but the only way to ensure persistence of absence of the breathing reflex is observation over a time period. Although arbitrary, a ten-minute apnea test seems both reasonable and, with a careful oxygenation technique, safe. ${ }^{16,17}$

All the minimum criteria for NDD must be met in the absence of confounding factors. CCDT recommendations make a non-exhaustive list of possible confounding factors, including shock, hypothermia, severe metabolic disorders capable of causing a potentially reversible coma, various severe metabolic abnormalities, peripheral nerve or muscle dysfunction, and drug intoxications. ${ }^{7}$ Others, such as a major facial trauma and high cervical spine injury, could be added to the list. The CCDT cautions examiners "to review confounding issues in the context of the primary etiology and examination. Clinical judgement is the deciding factor."7 Unfortunately, physician clinical judgement may show a high inter-individual variability, opening the door to mishaps. Identification of confounding factors may be challenging, and physicians have to be most vigilant in their systematic exclusion. The presence of any confounding factor precludes the clinical diagnosis of brain death. Signs of residual brain perfusion of the CT scan (such as the first case reported by Roberts) ${ }^{8}$ or intracranial flow velocity on transcranial Doppler should prompt suspicion of residual brain function. In such instances, physicians should be even more careful and should wait an observation period of a few hours in order to ascertain irreversibility before proceeding with NDD. ${ }^{15}$ It must be stressed that all of the minimum criteria for NDD must be met in order to make a medically, ethically, and legally valid declaration of death. If any of the minimum criteria cannot be evaluated adequately or if any confounding factor is present, a brain blood flow test is required for NDD. ${ }^{7,18}$ Absence of brain blood flow, which is similar to a physiologic decapitation, is an absolute and irreversible confirmation of whole brain death ${ }^{19}$ This criterion is more stringent than the clinical determination of brain stem death, where, in rare cases, residual flow might exist without invalidating the diagnosis of death. This double standard for the definition of brain death also generates confusion.

Clearly, the flawless application of the clinical criteria for NDD is not that simple and requires considerable expertise. On that matter, CCDT recommendations are limited to the requirement of a valid licensure for independent medical practice and "skill and knowledge in the management of patients with severe brain injury and in NDD." The latter is quite vague. Knowledge of clinical criteria for NDD and expertise in their application have been shown to be poor among health professionals. ${ }^{20-22}$ The report of Roberts shows that board-certified physicians with experience in the field of organ donation can also experience challenges with the application of current guidelines. ${ }^{8}$ Would better-defined guidelines help to prevent such incidents? Should the ability to make NDD be limited to physicians whose competency has been assessed by organ donation organizations?

Finally, the most important element of the whole process of NDD is irreversibility. On medical grounds, death is irreversible by definition. Irreversibility in the NDD refers to persistent absence over time of clinical signs of consciousness and brain stem function. The only way to ensure the persistence of the clinical criteria for NDD is to wait for a certain time interval before repeating the clinical examination. ${ }^{19}$ Previous Canadian guidelines for NDD required re-evaluation at a different time to ensure persistence of absence of brain function. ${ }^{4}$ Guidelines of the American Academy of Neurology also require a repeat clinical examination. ${ }^{3}$ However, current CCDT recommendations 
do not require a repeat clinical examination at a different time period. ${ }^{7}$ Moreover, if provincial law requires examination by a second physician for the purpose of transplantation, CCDT recommends no fixed time interval between the two examinations and even allows them to be done at the same time (with the exception of infants). ${ }^{7}$ Obviously, the duration of the time interval would have to be set arbitrarily, but six to $12 \mathrm{hr}$ between the two neurologic examinations seems reasonable and would satisfy the assessment of irreversibility. ${ }^{3,19}$

Physicians are not allowed to err when diagnosing death, even less when organ donation is contemplated. In this era of an ever-widening gap between the number of patients on transplantation wait lists and the number of organs available, it is commendable to try to increase the number of organ donors by promoting donation. However, we must remain very mindful not to jeopardize the whole organ donation and transplantation system by taking shortcuts with NDD. The trust of patients, families, and the general public in the organ donation process lies on their confidence that we will strictly respect the Dead Donor Rule and wait until death is confirmed before organ procurement. ${ }^{19}$ Moreover, there is now an alternative to the traditional heart-beating organ donation for patients who are not brain dead, although suffering a catastrophic brain injury for whom life support withholding is planned-organ donation after cardiocirculatory death. ${ }^{23}$

We are left with two possible conclusions: either sole clinical criteria are inadequate for diagnosis of brain death and a brain blood flow test should be mandatory for NDD, or clinical determination of brain death is adequate but the definition of the criteria must be strengthened and clinical NDD should be restricted to experienced practitioners in order to maintain the mandatory high specificity for NDD. While the former conclusion has the virtue of adding one more step before confirming the diagnosis, it may be excessive. Brain death remains primarily a clinical determination. ${ }^{7,18}$ Brain blood flow tests do not replace but rather complement clinical NDD when some of the minimum criteria are missing, when confounding factors are present, or when physicians or families have discomfort or uncertainty with the diagnosis. We believe that NDD can be properly reached in most cases using clinical criteria. However, seamless guidelines and expert knowledge in their application are mandatory. The problems reported by Roberts ${ }^{8}$ and observed by others in the field suggest that current CCDT recommendations for NDD have important gaps that need to be corrected. As anesthesiologists, intensivists, neurologists, or neurosurgeons, our first duty is to look after the best interests of our patient. Accurate diagnosis of death is part of it.

\section{Défis avec le diagnostic de la mort cérébrale}

Bien que les zones d'incertitude fassent partie de la pratique de la médecine clinique, le décès est un diagnostic sur lequel les médecins ne peuvent se permettre de se tromper. Le concept de mort cérébrale, qui a pour origine les critères de Harvard, ${ }^{1}$ a permis l'élaboration de programmes de greffes d'organes cadavériques. La définition de la mort cérébrale et les critères du diagnostic de décès neurologique (DDN) ont évolué au fil des ans. ${ }^{2-4}$ La loi américaine sur la détermination de la mort (Universal Determination of Death Act - UDDA) donne une définition de la mort mais remet le processus de diagnostic aux normes médicales acceptées (qu'elles soient nationales, provinciales ou locales), ce qui peut entraîner une variabilité considérable. ${ }^{2,5}$ En avril 2003, en réponse à un besoin perçu dans la communauté des soins critiques et médicale générale, le Conseil canadien pour le don et la transplantation (CCDT) a tenu un forum national afin de réévaluer le processus de détermination de la mort cérébrale et de proposer des recommandations mises à jour. ${ }^{6}$ Ce sont ces directives qui sont actuellement utilisées par les organismes de dons d'organes et de greffes partout au pays. ${ }^{7}$ Les recommandations du CCDT ont l'avantage d'avoir uniformisé le processus de DDN. Cependant, nombre de ces recommandations sont quelque peu vagues et ouvrent la voie au jugement clinique et à l'interprétation-une situation fort indésirable lorsqu'un don d'organe potentiel est envisagé.

Dans ce numéro du Journal, Roberts et coll. rapportent deux cas où des médecins expérimentés ont rencontré certaines difficultés dans le DDN clinique. ${ }^{8}$ Dans les deux cas, il semble que plusieurs des critères de DDN aient été mal interprétés ou négligés. Bien que généralement peu rapportés dans la littérature médicale, ce type d'incidents survient plus souvent qu'on ne le pense. Cette constatation est troublante. L'existence même de programmes de dons et de transplantation d'organes cadavériques s'appuie sur la règle du donneur décédé (Dead Donor Rule), laquelle stipule que le décès d'un patient doit être confirmé avant toute tentative de préservation ou de récolte d'organes à des fins de transplantation. ${ }^{9}$ Avant qu'un patient ne meure, les principes déontologiques dictent que les soins médicaux doivent être concentrés exclusivement sur le meilleur intérêt de ce patient et ce, même dans les situations où le patient peut être perçu comme «quasiment mort». La mort cérébrale est un concept complexe que le grand public a beaucoup de peine à comprendre. ${ }^{10,11}$ Les patients et leurs familles font confiance à leur médecin traitant. ${ }^{12}$ Dans les cas de dons d'organes, ils sont confiants que leurs 
médecins obéiront rigoureusement à la règle du donneur décédé. En conséquence, le processus de DDN doit impérativement être infaillible.

Les recommandations du CCDT définissent le décès neurologique comme la perte irréversible de toutes les fonctions du tronc cérébral. ${ }^{7}$ Les critères minimums pour un DDN clinique sont : 1) une étiologie établie pouvant mener au décès neurologique en l'absence de conditions réversibles pouvant y ressembler; 2) un coma profond sans réflexe et une absence bilatérale de réflexes moteurs, à l'exception des réflexes spinaux; 3) l'absence d'activité du tronc cérébral définie par l'absence du réflexe de déglutition, du réflexe de la toux, et l'absence bilatérale du réflexe cornéen, de réponses pupillaires à la lumière (avec pupilles de taille moyenne ou en mydriase), et de réflexes vestibulo-oculaires; 4) l'absence d'effort respiratoire confirmée par le test d'apnée; et 5) l'absence de facteurs confondants. ${ }^{7}$ Par minimum, on entend que tous ces critères doivent au moins être présents avant de pouvoir poser un DDN.

Le premier critère peut porter à confusion. Une étiologie «pouvant mener » au décès neurologique n'est pas à confondre avec une étiologie «suffisante pour expliquer » l'absence de signes cliniques de fonction cérébrale. Par exemple, un traumatisme cérébral constitue une étiologie pouvant mener à la mort cérébrale, mais il va sans dire que tous les traumatismes cérébraux ne se concluent pas par un décès neurologique. En effet, certains patients survivent avec un bon devenir fonctionnel à une lésion cérébrale aiguë grave qui aurait pu résulter en mort cérébrale. $^{13,14}$ C'est pourquoi les recommandations du CCDT précisent que «Il doit y avoir des preuves cliniques ou neuro-radiologiques formelles d'un accident aigu du système nerveux central (SNC) menant à la perte irréversible de la fonction neurologique ». ${ }^{7}$ Il doit y avoir une concordance absolue entre l'entité diagnostique provoquant la lésion cérébrale, sa gravité, et l'état neurologique clinique du patient. À ce titre, les examens diagnostiques de neuro-imagerie (par ex., les examens de tomodensitométrie, la résonance magnétique) sont très utiles, et il est crucial d'en faire une interprétation minutieuse. S'il existe un doute quelconque quant à l'étiologie ou à sa concordance avec l'état clinique, les médecins ne devraient alors pas procéder au DDN.

Il peut également y avoir confusion en ce qui touche à l'interprétation et la réalisation du test d'apnée. Un retour spontané de la respiration a été observé après un test d'apnée positif. ${ }^{8}$ Un test d'apnée positif n'est pas suffisant en soi pour diagnostiquer le décès neurologique. Il s'agit plutôt de la dernière étape dans l'évaluation de la fonction du tronc cérébral, et ce test ne devrait être réalisé qu'après confirmation de l'absence de tous les autres réflexes du tronc cérébral et après que tous les autres critères de DDN ont été confirmés. Il existe de nombreuses publications qui traitent de la technique, de la sécurité et des complications liées au test d'apnée. ${ }^{15-17}$ Un test d'apnée positif est défini comme l'absence d'effort respiratoire après la déconnexion $\mathrm{du}$ respirateur, accompagnée d'une $\mathrm{PaCO}_{2}$ s'élevant à plus de $60 \mathrm{mmHg}$ (et $\geq 20 \mathrm{mmHg}$ au-dessus du niveau avant le test d'apnée) et un $\mathrm{pH}$ inférieur à 7,28 . $^{7}$ Malheureusement, les lignes directrices ne spécifient pas la durée d'observation du patient. Il est vrai que nous ne savons pas combien de temps doit durer le test d'hypercapnie pour garantir l'absence de fonction centrale respiratoire, mais la seule façon de garantir que l'absence du réflexe respiratoire perdure est l'observation prolongée. Bien qu'arbitraire, un test d'apnée de dix minutes semble à la fois raisonnable et, s'il est réalisé avec technique d'oxygénation prudente, sécuritaire. ${ }^{16,17}$

Tous les critères minimums de DDN doivent être remplis en l'absence de facteur confondant. Les recommandations du CCDT dressent une liste non exhaustive des facteurs confondants possibles qui comprend le choc, l'hypothermie, les troubles métaboliques graves qui peuvent provoquer un coma potentiellement réversible, diverses anomalies métaboliques graves, le dysfonctionnement des nerfs périphériques ou musculaire et les intoxications médicamenteuses. ${ }^{7}$ D'autres facteurs, comme par exemple un traumatisme facial majeur ou une lésion haute de la colonne cervicale, pourraient s'ajouter à cette liste. Le CCDT demande aux médecins « d'évaluer l'importance de chaque facteur confondant dans le contexte de l'étiologie primaire et de l'évaluation clinique. (...) Le facteur décisif demeure le jugement clinique. " ${ }^{7}$ Malheureusement, le jugement clinique d'un médecin peut avoir une grande variabilité inter-individuelle, ce qui ouvre la voie à des incidents. L'identification des facteurs confondants peut être ardue, et les médecins doivent être très vigilants dans leur exclusion méthodique. La présence de tout facteur confondant exclut le diagnostic clinique de mort cérébrale. Les signes de perfusion cérébrale résiduelle au tomodensitogramme (comme dans le premier cas rapporté par Roberts $)^{8}$ ou la vitesse du débit intra-crânien mesurée par Doppler transcrânien devraient éveiller des soupçons quant à une fonction cérébrale résiduelle. Dans de tels cas, les médecins devraient faire encore plus attention et devraient prolonger la période d'observation à quelques heures de façon à confirmer l'irréversibilité avant de procéder au DDN. ${ }^{15}$ Il convient de souligner que tous les critères minimums de DDN doivent être remplis avant de faire une déclaration de décès qui soit valide d'un point de vue médical, déontologique et légal. Si l'un des critères minimums ne peut être évalué de façon appropriée ou s'il existe un quelconque facteur confondant, il faut procéder à une évaluation de la circulation sanguine cérébrale pour poser un DDN. ${ }^{7,18}$ L'absence de débit sanguin cérébral, 
semblable à une 'décapitation physiologique', est une confirmation absolue et irréversible de mort cérébrale complète. ${ }^{19} \mathrm{Ce}$ critère est plus rigoureux que le diagnostic clinique de mort du tronc cérébral où, dans de rares cas, un débit résiduel peut exister sans infirmer le diagnostic de la mort. Ce double standard dans la définition de la mort cérébrale crée également de la confusion.

Il est clair que l'application sans erreur des critères cliniques de DDN n'est pas si simple et requiert une grande expertise. À ce sujet, les recommandations du CCDT se limitent à l'exigence d'un permis d'exercice valide de pratique médicale indépendante et «les connaissances et les compétences requises pour la prise en charge de patients souffrant d'une lésion cérébrale grave et pour le $D D N .{ }^{7} \mathrm{La}$ seconde exigence est assez floue. Il a été démontré que la connaissance des critères cliniques pour le DDN et l'expertise dans leur application étaient faibles parmi les professionnels de la santé. ${ }^{20-22}$ Le compte-rendu de Roberts montre que des médecins spécialistes expérimentés dans le domaine des dons d'organe peuvent également être confrontés à des difficultés quant à l'application des directives actuelles. ${ }^{8}$ Si l'on disposait de directives plus claires, serait-il possible de prévenir de tels incidents? Devrait-on limiter le droit de poser un DDN aux médecins dont les compétences ont été évaluées par les organismes de dons d'organes?

Enfin, l'élément le plus important du processus de DDN est l'irréversibilité. Dans le domaine de la médecine, la mort est, par définition, irréversible. Quand on parle d'irréversibilité dans le DDN, on évoque l'absence permanente pendant une période prolongée de signes cliniques de conscience et de fonctions du tronc cérébral. La seule façon de garantir la permanence des critères cliniques de DDN est d'attendre un certain temps avant de refaire l'examen clinique. ${ }^{19}$ Les anciennes directives canadiennes concernant le DDN exigeaient une réévaluation à un moment différent afin de garantir la permanence de l'absence de fonction cérébrale. ${ }^{4}$ Les directives de l'Académie américaine de neurologie (American Academy of Neurology) exigent également une répétition de l'examen clinique. ${ }^{3}$ Les recommandations actuelles du CCDT ne requièrent toutefois pas de répétition de l'examen clinique à un moment différent. ${ }^{7}$ Qui plus est, si la loi provinciale exige un examen par un deuxième médecin à des fins de transplantation, le CCDT ne fait pas de recommandation quant à un intervalle de temps fixe entre les deux examens; il permet même que les deux examens soient réalisés simultanément (sauf pour les nourrissons). ${ }^{7}$ Il est évident que la durée de l'intervalle entre deux examens serait fixée de façon arbitraire, mais une durée de six à douze heures entre deux examens neurologiques semble raisonnable et satisferait à l'évaluation de l'irréversibilité. 3,19
Les médecins n'ont pas droit à l'erreur lorsqu'ils posent un diagnostic de décès, et encore moins lorsqu'un don d'organes est envisagé. En ces temps où le fossé entre le nombre de patients sur des listes d'attente de transplantation et le nombre d'organes disponibles ne cesse de s'élargir, il est louable d'essayer d'augmenter le nombre de donneurs d'organes en encourageant les dons. Nous devons cependant faire très attention à ne pas mettre en péril tout le système de dons et de transplantation d'organes. La confiance des patients, des familles et du grand public dans le processus de dons d'organes se fonde sur leur assurance que nous respecterons rigoureusement la règle du donneur décédé et attendrons que la mort soit confirmée avant de débuter le processus de prélèvement d'organes. ${ }^{19}$ De plus, il existe désormais une alternative au don d'organes traditionnel à cœur battant pour les patients qui ne sont pas en mort cérébrale, mais qui souffrent d'une lésion cérébrale catastrophique et chez qui on ne prévoit pas de maintenir les fonctions vitales : le don d'organes après décès cardiocirculatoire. ${ }^{23}$

Nous nous retrouvons avec deux conclusions possibles: soit les critères cliniques seuls ne suffisent pas pour poser un diagnostic de mort cérébrale et une évaluation de la sanguine cérébrale devrait être obligatoire pour un DDN, soit le diagnostic clinique de mort cérébrale est adéquat mais la définition des critères doit être renforcée et le DDN clinique devrait être restreint aux praticiens expérimentés afin de maintenir la spécificité élevée obligatoire du DDN. Alors que la première conclusion présente l'avantage d'ajouter une étape supplémentaire avant la confirmation du diagnostic, elle pourrait être excessive. La mort cérébrale demeure avant tout un diagnostic clinique. ${ }^{7,18}$ Les examens de la circulation sanguine cérébrale ne remplacent pas, mais plutôt complètent un DDN clinique lorsque certains des critères minimums font défaut, lorsqu'il y a des facteurs confondants, ou lorsque les médecins ou les familles éprouvent un malaise ou de l'incertitude face au diagnostic. Nous sommes d'avis qu'un DDN peut être correctement posé dans la plupart des cas sur la base des critères cliniques. Cependant, pour ce faire, des directives qui ne laissent place à aucune interprétation et une connaissance experte de leur application sont de mise. Les problèmes rapportés par Roberts $^{8}$ et ceux observés par d'autres dans ce domaine laissent à penser que les recommandations actuelles du CCDT sur le DDN comportent d'importantes lacunes qu'il convient de corriger. En tant qu'anesthésiologistes, intensivistes, neurologues, ou neurochirurgiens, notre devoir premier est de défendre les meilleurs intérêts de notre patient. Diagnostiquer correctement le décès en fait partie.

Disclosure Dr Brochu is Chair of the Medical Ethics Committee at the Centre hospitalier affilié universitaire de Québec. The authors 
have no financial conflict of interest pertaining to the subject of this manuscript.

Déclaration Le Dr Brochu est président du Comité d'éthique médicale au Centre hospitalier affilié universitaire de Québec. Les auteurs n'ont aucun conflit financier en lien avec le sujet de ce manuscrit.

\section{References}

1. Anonymous. A definition of irreversible coma. Report of the Ad Hoc Committee of the Harvard Medical School to Examine the Definition of Brain Death. JAMA 1968; 205: 337-40.

2. Anonymous. Guidelines for the determination of death. Report of the medical consultants on the diagnosis of death to the President's Commission for the Study of Ethical Problems in Medicine and Biomedical and Behavioral Research. JAMA 1981; 246: 2184-6.

3. Anonymous. Practice parameters for determining brain death in adults (summary statement). The Quality Standards Subcommittee of the American Academy of Neurology. Neurology 1995; 45: 1012-4.

4. Anonymous. Guidelines for the diagnosis of brain death. Canadian Neurocritical Care Group. Can J Neurol Sci 1999; 26: 64-6.

5. Greer DM, Varelas PN, Haque S, Wijdicks EF. Variability of brain death determination guidelines in leading US neurologic institutions. Neurology 2008; 70: 284-9.

6. Shemie SD, Doig C, Belitsky $P$. Advancing toward a modern death: the path from severe brain injury to neurological determination of death. CMAJ 2003; 168: 993-5.

7. Shemie $S D$, Doig $C$, Dickens $B$, et al. Severe brain injury to neurological determination of death: Canadian forum recommendations. CMAJ 2006; 174: S1-13.

8. Roberts DJ, MacCulloch KA, Versnick EJ, Hall RI. Should ancillary brain blood flow analyses play a larger role in the neurological determination of death? Can J Anesth 2010; 57. DOI: $10.1007 / \mathrm{s} 12630-010-9359-4$.

9. Robertson JA. The dead donor rule. Hastings Cent Rep 1999; 29: 6-14.
10. Sanner M. A comparison of public attitudes toward autopsy, organ donation, and anatomic dissection. A Swedish survey. JAMA 1994; 271: 284-8.

11. Doig CJ, Burgess E. Brain death: resolving inconsistencies in the ethical declaration of death. Can J Anesth 2003; 50: 725-31.

12. Heyland DK, Dodek $P$, Rocker $G$, et al. What matters most in end-of-life care: perceptions of seriously ill patients and their family members. CMAJ 2006; 174: 627-33.

13. Skoglund TS, Nellgaird B. Long-time outcome after transient transtentorial herniation in patients with traumatic brain injury. Acta Anaesthesiol Scand 2005; 49: 337-40.

14. Chamoun RB, Robertson CS, Gopinath SP. Outcome in patients with blunt head trauma and a Glasgow Coma Scale score of 3 at presentation. J Neurosurg 2009; 111: 683-7.

15. Wijdicks $E F$. Clinical diagnosis and confirmatory testing of brain death in adults. In: Wijdicks EF, editor. Brain Death. Philadelphia: Lippincott Williams \& Wilkins; 2001. p. 61-90.

16. Levesque $S$, Lessard $M R$, Nicole PC, et al. Efficacy of a T-piece system and a continuous positive airway pressure system for apnea testing in the diagnosis of brain death. Crit Care Med 2006; 34: 2213-6.

17. Wijdicks EF, Rabinstein AA, Manno EM, Atkinson JD. Pronouncing brain death: contemporary practice and safety of the apnea test. Neurology 2008; 71: 1240-4.

18. Shemie SD, Lee D, Sharpe M, Tampieri D, Young B, Canadian Critical Care Society. Brain blood flow in the neurological determination of death: Canadian expert report. Can J Neurol Sci 2008; 35: 140-5.

19. Bernat JL. The concept and practice of brain death. Prog Brain Res 2005; 150: 369-79.

20. Youngner SJ, Landefeld CS, Coulton CJ, Juknialis BW, Leary M. 'Brain death' and organ retrieval. A cross-sectional survey of knowledge and concepts among health professionals. JAMA 1989; 261: 2205-10.

21. Mejia RE, Pollack MM. Variability in brain death determination practices in children. JAMA 1995; 274: 550-3.

22. Wang $M Y$, Wallace $P$, Gruen JP. Brain death documentation: analysis and issues. Neurosurgery 2002; 51: 731-5.

23. Shemie SD, Baker AJ, Knoll G, et al. National recommendations for donation after cardiocirculatory death in Canada: donation after cardiocirculatory death in Canada. CMAJ 2006; 175: S1-24. 